\begin{tabular}{lc} 
Sharif University of Technology & Scientia Iranica \\
SCIENTIA & Transactions F: Nanotechnology \\
I RAN ICA & http://scientiairanica.sharif.edu \\
\hline
\end{tabular}

\title{
Electrospinning of smart thermochromic nanofibers as
}

\section{sensors}

\author{
N. Eslahi ${ }^{a, *}$, T. Fatemia ${ }^{a}$, M. Varsei ${ }^{a}$, and S. Bazgir ${ }^{b}$ \\ a. Department of Textile Engineering, Science and Research Branch, Islamic Azad University, Tehran, Iran. \\ b. Department of Petroleum and Chemical Engineering, Science and Research Branch, Islamic Azad University, Tehran, Iran.
}

Received 29 March 2020; received in revised form 16 August 2020; accepted 21 September 2020

\section{KEYWORDS}

Thermochromic;

PDA;

Nanofibers;

Electrospinning;

Sensors.

\begin{abstract}
Color-transition features of polydiacetylene (PDA) have gained attention in recent years owing to its potential use in a wide range of sensors. This paper investigates electrospinning of PDA and polyvinylpyrolidone (PVP) to develop thermochromic nanofibrous composites. Polymer mixtures at different mass ratios and concentrations were electrospun and characterized afterwards. SEM results showed successful electrospinning of continuous and bead-free nanofibers with different diameters depending on the mass ratio of PVP to PDA. The obtained fibrous mats were then photo-polymerized under UV irradiation, which led to generation of a blue color showing the self-assembly of diacetylene monomers. The colorimetric transition of the fibers was also investigated and a color change from blue to red occurred by heating the fibers up to $80^{\circ} \mathrm{C}$ because of the conformational alterations in the PDA molecules. FTIR and DSC analysis validated these findings as well. As a result, the fabricated nanofibrous composites are potentially appropriate candidates for sensing applications.
\end{abstract}

(C) 2020 Sharif University of Technology. All rights reserved.

\section{Introduction}

Recent advances in nanotechnology have led to an upsurge of interest in the development of sensors with enhanced sensitivity and specificity [1]. Various electrospun polymer nanofibers have been developed as sensors owing to their intrinsically high aspect ratio, surface functionality, low cost, and simple fabrication $[2,3]$. Electrospun nanofibers have been employed in a wide range of detection systems such as colorimetric, biological, and electrochemical applications [4]. In particular, interest has grown in colorimetric sensors

\footnotetext{
*. Corresponding author.

E-mail address: niloofar.eslahi@srbiau.ac.ir (N. Eslahi)
}

doi: $10.24200 /$ sci. 2020.55714 .4369 owing to their portability, rapid response, accuracy, and cost-effectiveness [5].

Conjugated polymers have recently gained attention in sensor development since they impart easy signal analysis, color transitions and facile diagnosis [6]. Besides, owing to the electrical conductivity along the polymer backbone, conjugated polymers can provide a more sensitive response than small molecules in various sensing technologies [7]. Polydiacetylene (PDA) is an interesting conjugated polymer due to its unique blue-to-red color transition in response to external stimuli (such as heat, $\mathrm{pH}$, and organic solvents), which can be easily observed by the naked eye [8]. PDA can be simply synthesized by polymerizing diacetylene monomers under ultraviolet or gamma irradiation without the need for chemical initiators or catalysts [9]. Thermochromism is a phenomenon in which a material switches color in response to a change in tempera- 
ture [10]. The colorimetric transition temperatures of PDAs correspond with the melting points of the diacetylene monomers which usually occurs above room temperature (typically $>40^{\circ} \mathrm{C}$ ) [11]. PDAs in various forms such as films, powders, coatings, and fibers have been studied and used for sensing chemical or biological targets $[12,13]$.

For production of ultrasensitive sensors, electrospinning is a promising fabrication technique for a providing high surface area [14]. Electro spinning has proven an efficient method to produce polymeric fibers having a porous structure and good interconnectivity [15]. It can be employed to design selfassembled nanostructures with adjustable features for developing multifunctional devices [2]. PDA electrospun nanofibers are particularly promising for optical sensing owing to simple functionalization, facile synthesis, and the adaptability to enable colorimetric or fluorescence signaling $[16,17]$. Because of the high cost and low spinnability of PDA, this conjugated polymer has been mixed with other polymers to produce nanofibrous composites [9]. According to Yapor et al., PDA-containing nanofiber composites exhibited profounder colorimetric responses than those of the pristine PDA [5]. Jeon et al. functionalized diacetylene monomers with trimethyl amine (PCDADMEDA) and mixed them with Poly (Ethylene Oxide) (PEO) into electrospun fibers for potential diagnosis of $\mathrm{HCl}$ gas [18]. In another study, PDA was combined with PEO to produce electrospun nanofibers with optical sensing ability for detection of volatile organic compounds [16]. Moreover, Ali et al. fabricated PDApoly $\varepsilon$-caprolactone (PCL) electrospun fiber mats for colorimetric detection of fake gasoline [19].

In this study, PDA and polyvinylpyrolidone (PVP) were used for the first time to develop nanofiber composites via an electrospinning process. PVP is a synthetic polymer exhibiting proper physiological compatibility, low chemical toxicity, transparency, good solubility in most organic solvents, suitable spinnability, antibacterial activity, and the ability to interact with different materials [20,21]. Electrospun PVPbased fibers have also been applied in different chemical and biological sensing $[22,23]$. The aim of this study is to evaluate the feasible fabrication of PDA-PVP nanofibrous composites as potential sensors. The produced electrospun fibers were characterized afterwards by SEM, FTIR, and DSC analysis, and the colorimetric transition behavior was investigated by heating the produced fibers.

\section{Experimental}

\subsection{Materials}

10,12-pentacosadiynoic acid (PCDA, 98\%) as PDA monomer and polyvinylpyrolidone (PVP, MW 360000) were purchased from Sigma-Aldrich (USA). Ethanol and other chemicals were of analytical grade and obtained from Merck (Germany).

\subsection{Methods}

Mixture solutions of polymers in ethanol were prepared at two different mass ratios of PVP to PCDA (3:1 and $4: 1$ ) and at various polymer concentrations (4, 6, 8, and $10 \mathrm{wt} \%)$. The resulting solutions were then stirred overnight at room temperature and electrospun via an electrospinning apparatus (Labscale Electrospinning Unit, ESDP30, Iran). The polymer solution was fed into the horizontally aligned syringe and an aluminium foil was used for covering the collector. Several processing parameters were tested in order to optimize the electrospinning conditions and the optimal parameters were chosen as follows: feeding rate of $0.5 \mathrm{~mL} / \mathrm{h}$, applied voltage of $15 \mathrm{kV}$, and tip-to-collector distance of $15 \mathrm{~cm}$. The electrospun mats were dried and kept in the dark overnight before use.

Photopolymerization of PCDA was carried out by irradiatiing the fibers under $\mathrm{UV}_{\mathrm{c}}$ light (HPA Philips, 30 $\mathrm{W}$, Poland) for $5 \mathrm{~min}$. After irradiation with UV-light at $254 \mathrm{~nm}$, the color of the white colored electrospun fibers turned blue validating the polymerization of diacetylene monomers. The colorimetric transition of the fibers was investigated by heating them at different temperatures $\left(40,60\right.$ and $80^{\circ} \mathrm{C}$ ) for $15 \mathrm{~min}$ in an oven and the reflectance spectra were measured by a reflectance spectrophotometer (Varian Cary 500, Australia). The dimension and morphology of the fibers were explored using a scanning electron microscope (SEM, Philips XL30, USA) at a voltage of $25 \mathrm{kV}$ after sputter coating with gold. The average fiber diameter was measured for each sample (out of 30 random fibers) by employing image analysis software (Image J, National Institute of Health, Bethesda, MD). To study the chemical structure of the obtained composites, FTIR analysis was performed at $400-4000 \mathrm{~cm}^{-1}$ using a Thermo Nicolet FTIR spectrometer (NEXUS 870, USA). Differential scanning calorimetry (DSC 131 SETARAM, France) was also utilized to evaluate the thermal transitions of the fibers at a heating rate of $10^{\circ} \mathrm{C} / \mathrm{min}$ in a nitrogen atmosphere.

\section{Results and discussion}

The SEM images of the electrospun fibers together with their relative size distribution plots are presented in Figure 1. The fibrous mats resemble a spider web comprising of intermingled thin and thick fibers. Upon elongation of the electrically actuated polymer jet in the spinning region, the entanglement of polymer chains hampers them from disintegrating and beadfree, long, and continuous fibers are formed [24]. The fiber diameter varied depending on the polymers ratio 
$10 \%$

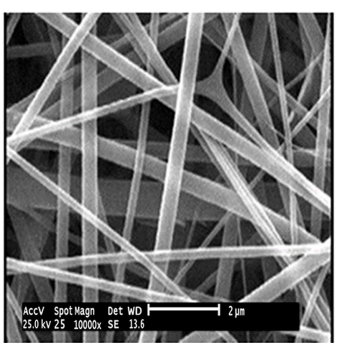

$3: 1$
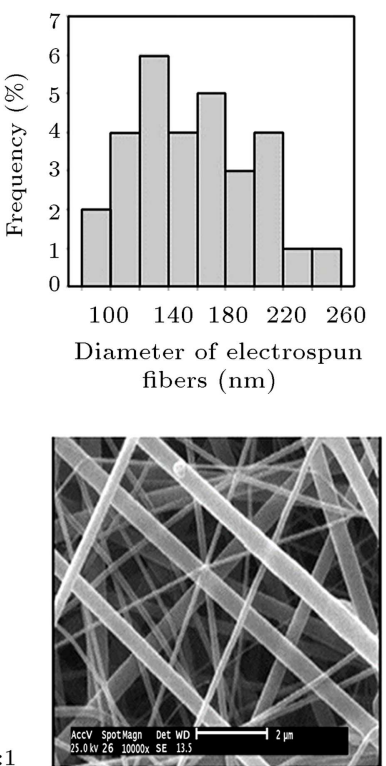

$4: 1$
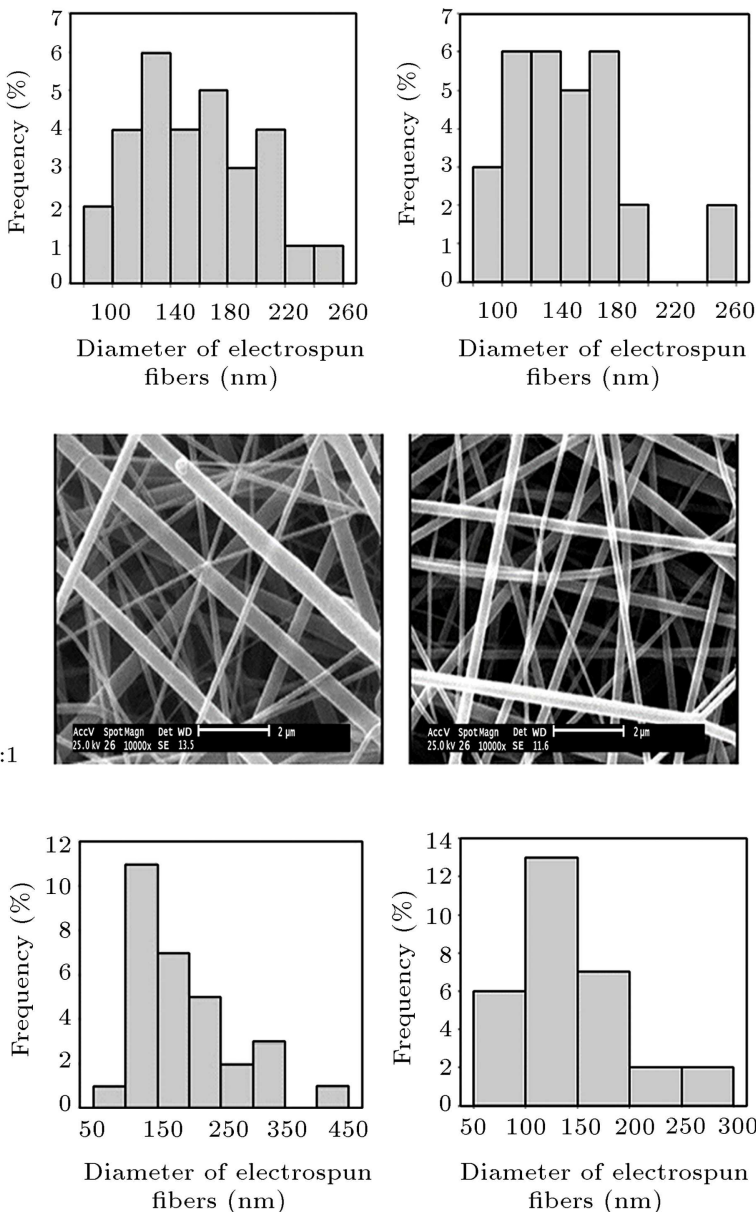

$8 \%$
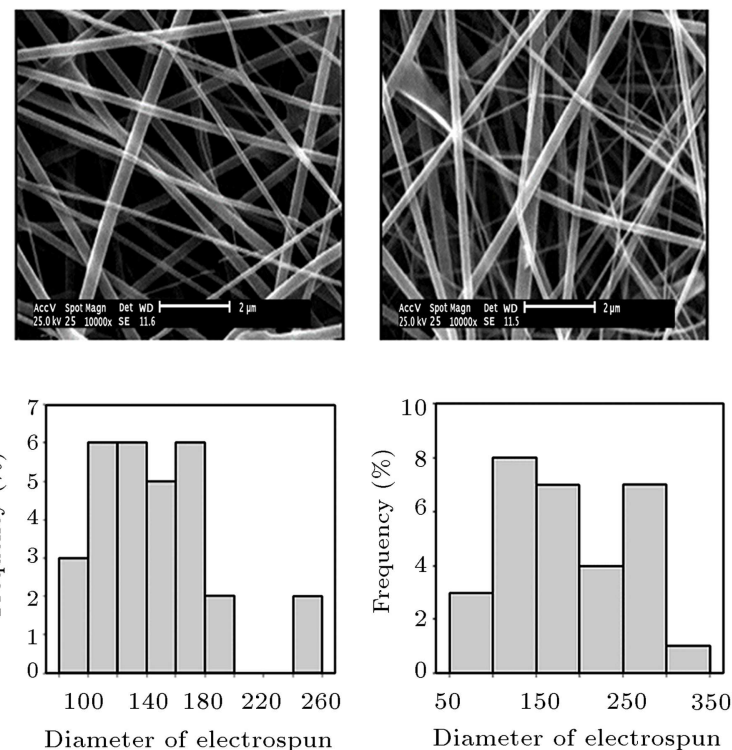

fibers $(\mathrm{nm})$

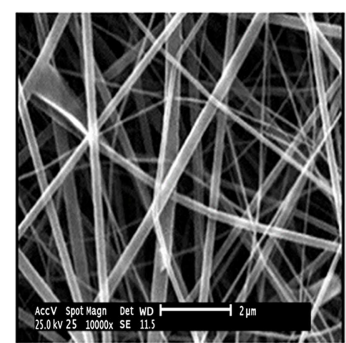

$4 \%$
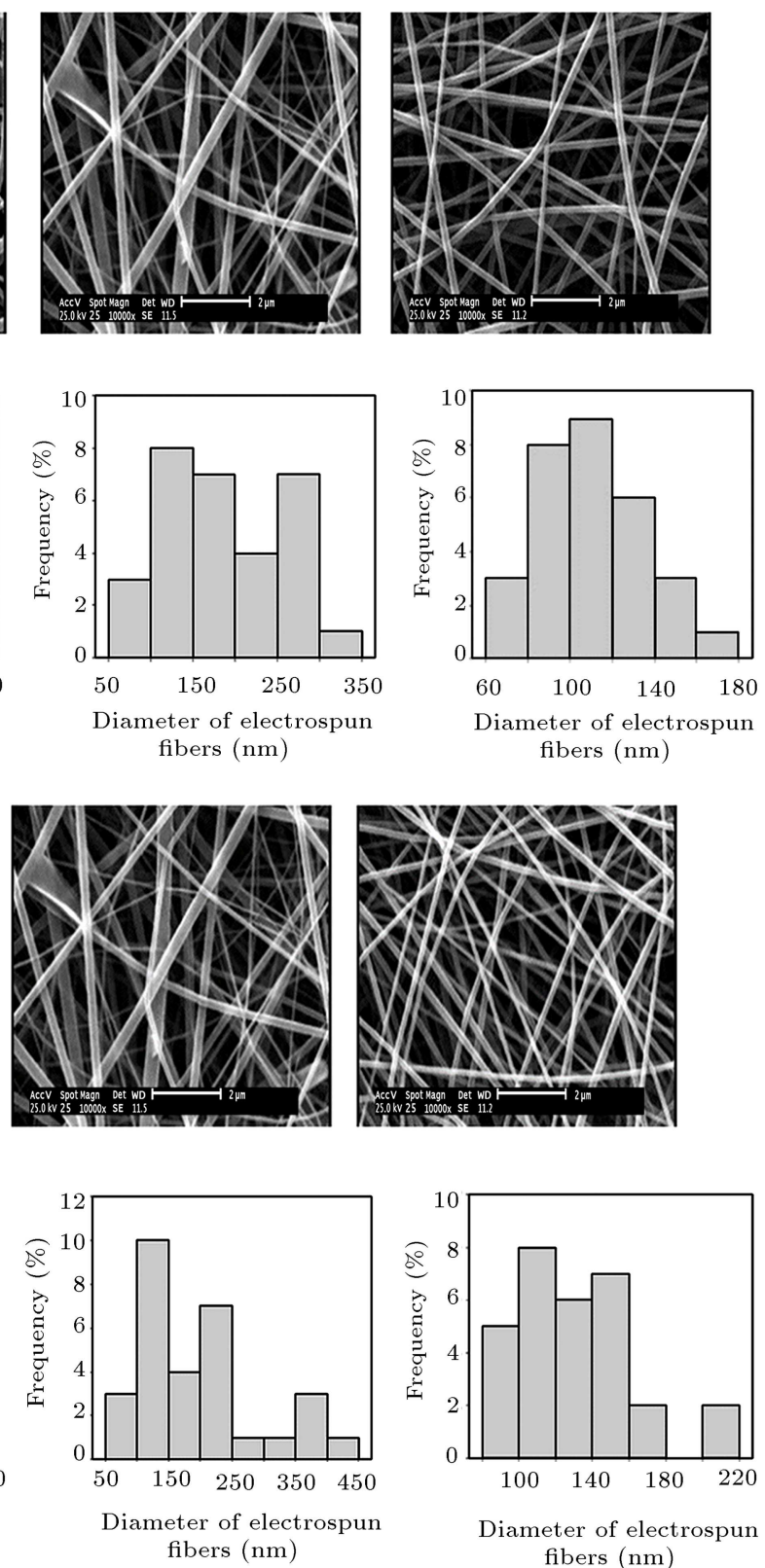

Figure 1. SEM images and fiber diameter distribution plots of electrospun mats at different polymer concentration and two mass ratios of PVP to PCDA.

and concentration. As can be seen, with decreasing polymer concentration, the average fiber diameter is declined owing to lower electrospinning force and results in a better stretching of fibers. Increasing polymer concentration inhibits fast changes in the Taylor cone, leading to formation of thick fibers [8].

Furthermore, the 3:1 mass ratio of PVP to PCDA promoted more uniform and thinner fibers at all concentrations, possibly owing to the suitable viscosity of PVP aiding fiber stretchability. Consequently, electrospun fibers at 3:1 mass ratio and $4 \%$ polymer concentration are selected as the optimum specimen having the minimum average fiber diameter.
During solvent evaporation and fiber formation in the electrospinning process, PCDA self-assembly occurred, since the attractive forces between the DA monomers were stronger than those between the PVP polymer and DA monomers [18]. Polymerization of PCDA was then conducted by irradiating the electrospun webs under $254 \mathrm{~nm} \mathrm{UV}$ light for $5 \mathrm{~min}$. A distinctive blue color after UV light irradiation confirmed successful photopolymerization in the fibers through conjugation, suggesting that the delocalized $\pi$ network and the conformational constraints within the polymer chain were preserved in the electrospinning procedure [16]. Besides, by heating the fibers up 
to $80^{\circ} \mathrm{C}$, a blue-to-red color transition occurred due to the twisting of the conjugated backbone of the PDA. In fact, changes in temperature decrease the conjugation length and expand the energy band gap in PDA structures [25]. It was found that changes in PDA side chain length as well as PVP molecular weight could influence the thermochromic behavior of PDAPVP nanocomposite films. There was a reduction in color-transition temperature with a shortening of the PDA alkyl tail and increasing PVP molecular weight owing to the diminished dispersion interactions in the nanocomposite [26].

The colorimetric transition of the electrospun fibers was assessed by a reflectance spectrophotometer. The obtained results in Figure 2 show a significant decline in the reflectance of the UV irradiated sample at $650 \mathrm{~nm}$ to $700 \mathrm{~nm}$ corresponding to the formation of a blue color. Moreover, no major morphological alterations were seen in the SEM images before and after UV-irradiation (data not shown). To better show the temperature sensitivity of the produced fibers, they were heated at three different temperatures and the resulting reflectance spectra were measured. Upon heating the fibers, a reflectance switch to a lower wavelength was observed, resulting in a red color. As can be seen, by increasing temperature from $25^{\circ} \mathrm{C}$ to $80^{\circ} \mathrm{C}$, the minimum reflectance wavelength $\left(\lambda_{\min }\right)$ shifts from $700 \mathrm{~nm}$ to $550 \mathrm{~nm}$ with an abrupt reduction in the whole reflectance, validating the blue-to-red color change by an increase in temperature. This phenomenon (thermochromism) is the main reason for various applications of PDA as sensors. The obtained results also revealed that the matrix polymer in the PVP-PDA fiber composite had no negative effect on the colorimetric transition of PDA. A literature review

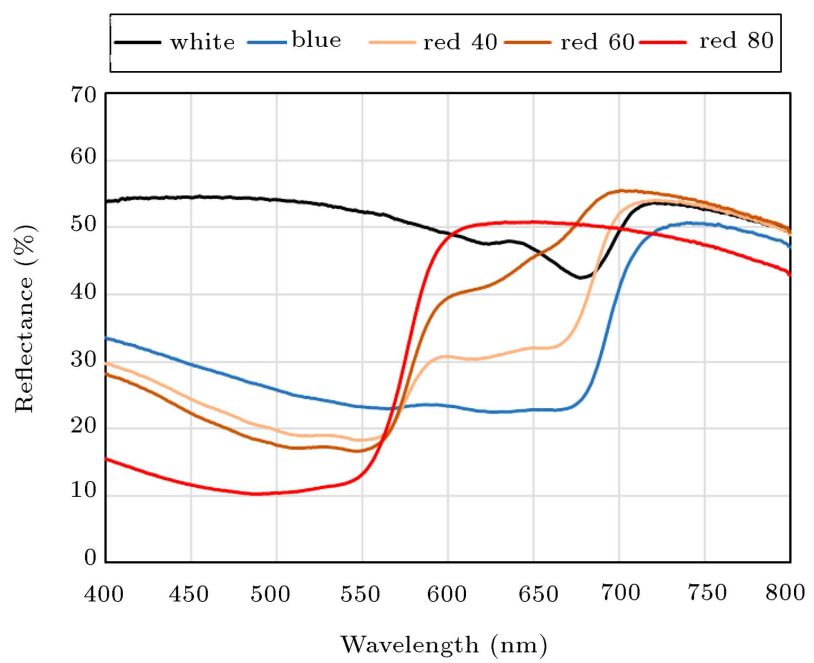

Figure 2. Reflectance spectra of electrospun fibers: White: before irradiation, blue: after UV irradiation, and red: after heating at 40,60 and $80^{\circ} \mathrm{C}$. showed that the colorimetric transition temperature of PDA is dependent on the melting point of the corresponding DA monomer indicating conformational changes in the macromolecule [11].

To examine any possible changes in the chemical structure upon UV irradiation and heating, FTIR spectra of the fibrous membranes were recorded. Figure 3 illustrates the characteristic functional groups of the employed polymers. A broad absorption peak at around $3500 \mathrm{~cm}^{-1}$ is associated with the stretching vibration of $\mathrm{O}-\mathrm{H}$ groups in $\mathrm{PDA}$. In addition, formation of hydrogen bonds between carboxylic groups of PDA and carbonyl group of PVP could occur in the composite [26]. The peak in the range of 3000$2800 \mathrm{~cm}^{-1}$ belongs to the $\mathrm{C}-\mathrm{H}$ stretching vibration of methylene groups. The observed peaks in $1675 \mathrm{~cm}^{-1}$ and $1281 \mathrm{~cm}^{-1}$ correspond to the $\mathrm{C}=\mathrm{O}$ stretching vibration of both polymers and $\mathrm{C}-\mathrm{N}$ stretching vibration of PVP, respectively [27]. The peak in $1433 \mathrm{~cm}^{-1}$ is also attributed to $\mathrm{CH}_{2}$ bending vibration. As can be seen, there is no significant differences between the samples before (white) and after UV irradiation (blue). However, after heating (red), the intensity of the peaks declined noticeably which might be due to the conformational variations in PDA disrupting the $\pi$ overlap and, as a result, changing the electronic configuration of the polymer [28].

The thermal transitions of the polymerized electrospun fibers were determined by DSC analysis, and the melting temperature $\left(T_{m}\right)$ and the corresponding enthalpy were calculated for each specimen. According to Figure 4, the UV-irradiated nanofibers (blue sample) show an endothermic peak $\left(T_{m}\right)$ at $74^{\circ} \mathrm{C}$ with enthalpy of $104.72 \mathrm{~J} / \mathrm{g}$. After heating (red sample), this peak shifted slightly to a lower temperature at $70^{\circ} \mathrm{C}$, while

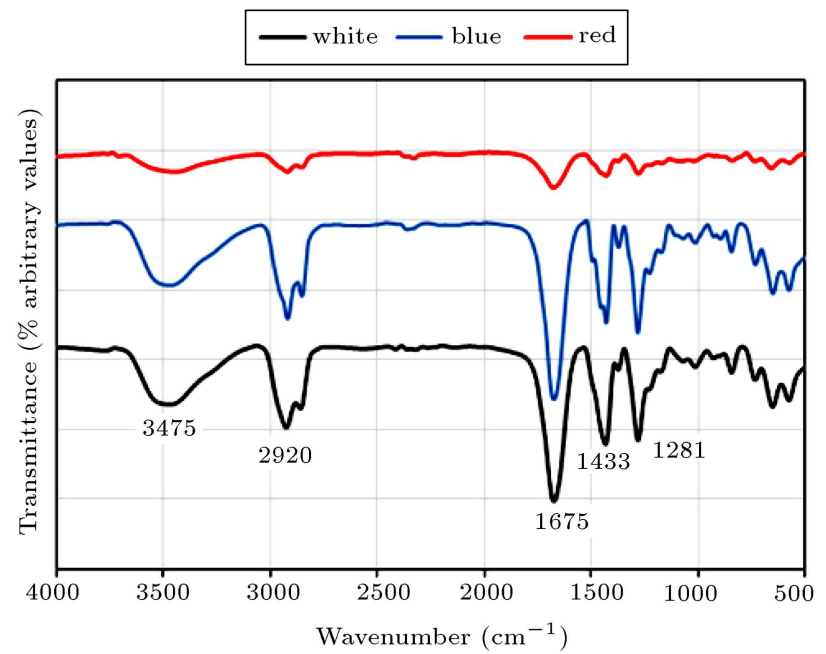

Figure 3. FTIR spectra of samples: White: before irradiation, blue: after UV irradiation, and red: after heating. 


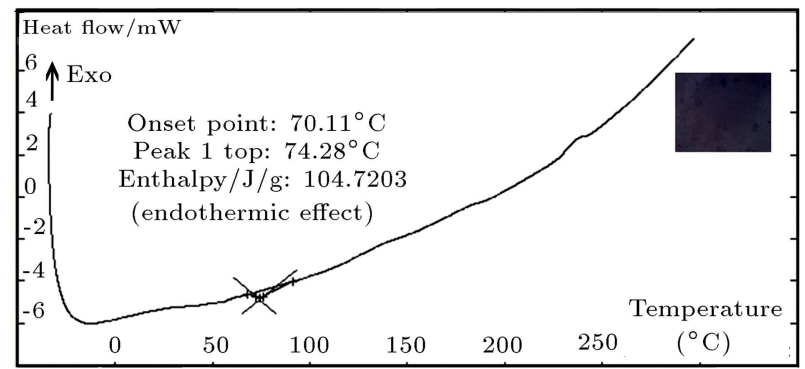

(a)

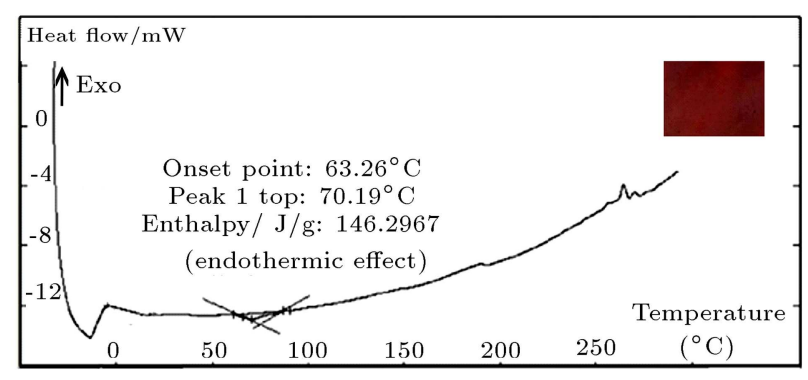

(b)

Figure 4. DSC plots of PVP-PDA fibers: (a) Blue and (b) red.

the enthalpy increased to $146.3 \mathrm{~J} / \mathrm{g}$. It is worth mentioning that the melting temperature appears at around $70^{\circ} \mathrm{C}$, which is similar to the color transition temperature from blue to red upon heating. The optical properties of PDA depend on the structural alignment of the polymer chains. With increasing temperature, C-C bond rotation is expedited and the planarity of the polymer confirmation is disrupted, resulting in color change [8].

\section{Conclusions}

Polydiacetylene (PDA) is an attractive conjugated material due to its unique thermochromism in response to external stimuli. Mixtures of PDA and PVP solutions were employed to develop nanofiber composites via a simple electrospinning process. The smooth and beadfree electrospun fibers were obtained and then photopolymerized using UV-light irritation. SEM images showed that the size and uniformity of the fibers were dependent on polymer concentration as well as their ratio in the mixture. The colorimetric transition was evaluated by a reflectance spectrophotometer and a color change from blue to red was observed upon heating, with a pronounced color switch above $60^{\circ} \mathrm{C}$. The FTIR and DSC analysis showed that the color transition of the electrospun fibers was due to the conformational changes in PDA macromolecules, implying that addition of Polyvinylpyrolidone (PVP) in the mixture did not influence the optical properties of PDA. Therefore, the fabricated mats could be used as thermochromic sensors.

\section{Acknowledgements}

We would like to express our gratitude to the members of the Iranian Academic Center for Education, Culture \& Research (Amirkabir University of Technology Branch).

\section{References}

1. Ding, B., Wang, M., Wang, X., Yu, J., and Sun, G. "Electrospun nanomaterials for ultrasensitive sensors", Mater. Today, 13(11), pp. 16-27 (2010).

2. Chinnappan, A., Baskar, C., Baskar, S., Ratheesh, G., and Ramakrishna, S. "An overview of electrospun nanofibers and their application in energy storage, sensors and wearable/flexible electronics", J. Mater. Chem. C., 5(48), pp. 12657-12673 (2017).

3. Wang, X., Li, Y., and Ding, B. "Electrospun Nanofiber-Based Sensors", In Electrospun Nanofibers for Energy and Environmental Applications (Ding, B., and $\mathrm{Yu}$, J. eds.), Springer, Berlin, Heidelberg, pp. 267297 (2014).

4. Sapountzi, E., Braiek, M., Chateaux, J.-F., JaffrezicRenault, N., and Lagarde, F. "Recent advances in electrospun nanofiber interfaces for biosensing devices", Sensors, 17(8), p. 1887 (2017).

5. Yapor, J.P., Alharby, A., Gentry-Weeks, C., Reynolds, M.M., Alam, A.K.M.M., and Li, Y.V. "Polydiacetylene nanofiber composites as a colorimetric sensor responding to escherichia coli and $\mathrm{pH}$ ", ACS Omega, 2(10), pp. 7334-7342 (2017).

6. Sun, X., Chen, T., Huang, S., Li, L., and Peng, H. "Chromatic polydiacetylene with novel sensitivity", Chem. Soc. Rev., 39(11), pp. 4244-4257 (2010).

7. Chen, X., Zhou, G., Peng, X., and Yoon, J. "Biosensors and chemosensors based on the optical responses of polydiacetylenes", Chem. Soc. Rev., 41(13), pp. 46104630 (2012).

8. Alam, A., Yapor, J.P., Reynolds, M.M., and Li, Y.V. "Study of polydiacetylene-poly (ethylene oxide) electrospun fibers used as biosensors", Materials, 9(3), p. 202 (2016).

9. Jaewon, Y., Young-Sik, J., and Jong-Man, K. "A combinatorial approach for colorimetric differentiation of organic solvents based on conjugated polymerembedded electrospun fibers", Adv. Funct. Mater., 19(2), pp. 209-214 (2009).

10. Shin, M.J., and Kim, J.-D. "Chromatic reversibility of multilayered polydiacetylene cast film", J. Ind. Eng. Chem., 35, pp. 211-216 (2016).

11. Park, I.S., Park, H.J., Jeong, W., Nam, J., Kang, Y., Shin, K., Chung, H., and Kim, J.-M. "Low temperature thermochromic polydiacetylenes: design, colorimetric properties, and nanofiber formation", Macromolecules, 49(4), pp. 1270-1278 (2016).

12. Lee, S., Kim, J.-Y., Chen, X., and Yoon, J. "Recent progress in stimuli-induced polydiacetylenes for 
sensing temperature, chemical and biological targets", Chem. Commun., 52(59), pp. 9178-9196 (2016).

13. Lebegue, E., Farre, C., Jose, C., Saulnier, J., Lagarde, F., Chevalier, Y., Chaix, C., and Jaffrezic-Renault, N. "Responsive polydiacetylene vesicles for biosensing microorganisms", Sensors, Basel, Switzerland, 18(2), p. 599 (2018).

14. Sapountzi, E., Braiek, M., Chateaux, J.F., JaffrezicRenault, N., and Lagarde, F. "Recent advances in electrospun nanofiber interfaces for biosensing devices", Sensors, Basel, Switzerland, 17(8), p. 1887 (2017).

15. Bhardwaj, N., and Kundu, S.C. "Electrospinning: a fascinating fiber fabrication technique", Biotechnol. $A d v$, 28(3), pp. 325-347 (2010).

16. Davis, B.W., Burris, A.J., Niamnont, N., Hare, C.D., Chen, C.-Y., Sukwattanasinitt, M., and Cheng, Q. "Dual-mode optical sensing of organic vapors and proteins with polydiacetylene (PDA)-embedded electrospun nanofibers", Langmuir, 30(31), pp. 9616-9622 (2014).

17. Lee, J., Balakrishnan, S., Cho, J., Jeon, S.-H., and Kim, J.-M. "Detection of adulterated gasoline using colorimetric organic microfibers", J. Mater. Chem., 21(8), pp. 2648-2655 (2011).

18. Jeon, H., Lee, J., Kim, M.H., and Yoon, J. "Polydiacetylene-based electrospun fibers for detection of $\mathrm{HCl}$ gas", Macromol. Rapid Commun., 33(11), pp. 972-976 (2012).

19. Ali, S., Ahmed, F., and Khatri, A. "Polycaprolactonepolydiacetylene electrospun fibers for colorimetric detection of fake gasoline", Mehran University Research Journal of Engineering \& Technology, 35(2), p. 287 (2016).

20. Reddy, V.R.T., Reddy, K.K., and Ravindra, S. "Effect of optimization parameters on the diameter of PVP fibers fabricated by electrospinning technique", International Conference on Advanced Nanomaterials \& Emerging Engineering Technologies, Chennai, India, pp. $733-736$ (2013).

21. Nasouri, K., Shoushtari, A.M., and Mojtahedi, M.R.M. "Effects of polymer/solvent systems on electrospun polyvinylpyrrolidone nanofiber morphology and diameter", Polym. Sci. Ser. A., 57(6), pp. 747755 (2015).

22. Matatagui, D., Fernández, M.J., Santos, J.P., Fontecha, J., Sayago, I., Horrillo, M.C., Grácia, I., and Cané, C. "Real-time characterization of electrospun PVP nanofibers as sensitive layer of a surface acoustic wave device for gas detection", J. Nanomater., 2014 p. 8 (2014).

23. Dai, M., Jin, S., and Nugen, S.R. "Water-soluble electrospun nanofibers as a method for on-chip reagent storage", Biosensors, 2(4), pp. 388-395 (2012).
24. Lukáš, D., Sarkar, A., Martinová, L., Vodsed'álková, K., Lubasová, D., Chaloupek, J., Pokorný, P., Mikeš, P., Chvojka, J., and Komárek, M. "Physical principles of electrospinning (electrospinning as a nanoscale technology of the twenty-first century)", Textile Progress, 41(2), pp. 59-140 (2009).

25. Jelinek, R. and Ritenberg, M. "Polydiacetylenes-recent molecular advances and applications", RSC Adv., 3(44), pp. 21192-21201 (2013).

26. Kamphan, A., Khanantong, C., Traiphol, N., and Traiphol, R. "Structural-thermochromic relationship of polydiacetylene (PDA)/polyvinylpyrrolidone (PVP) nanocomposites: Effects of PDA side chain length and PVP molecular weight", J. Ind. Eng. Chem., 46, pp. 130-138 (2017).

27. Huang, S., Zhou, L., Li, M.-C., Wu, Q., Kojima, Y., and Zhou, D. "Preparation and properties of electrospun poly (vinyl pyrrolidone)/cellulose nanocrystal/silver nanoparticle composite fibers", Materials, 9(7), p. 523 (2016).

28. Scoville, S.P. and Shirley, W.M. "Investigations of chromatic transformations of polydiacetylene with aromatic compounds", J. Appl. Polym. Sci., 120(5), pp. 2809-2820 (2011).

\section{Biographies}

Niloofar Eslahi completed BS (2007), MS (2009) and PhD (2013) degrees with the highest placing from the Textile Engineering Department (Textile Chemistry and Fiber Science) and admitted to the Center of Exceptional Talents at Amirkabir University of Technology (AUT, Iran). She received an Allameh post-doctoral fellowship (2014) from the Iran National Elites Foundation to carry out research at the Center for Nanostructured and Advanced Materials (CNAM) at the Department of Materials Science and Engineering, Sharif University of Technology (SUT). She was granted funding from the Iran National Science Foundation (INSF) for US Patent 14/641,460 in 2015 and from the Iran Patent Office for Provisional US Patent Application 62512018 in 2017. Currently, Dr. Eslahi is Assistant Professor in the Engineering Department at the Science and Research Branch of the Islamic Azad University of Tehran and a professional member of the Iran Nanotechnology Initiative Council (INIC), Iranian Textile Association of Science and Technology (ITAST), and Young Researchers and Elite Club. Her research interests are focused on medical textiles, smart biomaterials, electrospun nanofiber composites, and biocompatible nanomaterials for tissue engineering applications.

Tara Fatemi was an MS graduate student in the Department of the Textile Engineering, Science and Research Branch of the Islamic Azad University, Tehran, Iran in 2018. Her research interests include electrospun 
nanofibers, smart textiles and sensors.

Mehdi Varsei received MS (2004) and PhD (2012) degrees from Amirkabir University of Technology, and the Science and Research Branch of the Islamic Azad University, Tehran, Iran, respectively. He is currently President of ACECR, Amirkabir University of Technology Branch, and also Assistant Professor of Textile Engineering at the Science and Research Branch of the Islamic Azad University, Tehran. During the past 20 years, he has been a consultant for various apparel and textile industries and delivered numerous lectures, seminars and workshops for both academics and industry executives in the field. He is the author of three books, journal articles, peer-reviewed conference papers and book chapters. His expertise includes technical textiles, textile industry, smart textiles, textile management, textile reinforced concrete (TRC), fibre reinforced concrete (FRC) and composites.

Saeed Bazgir received MS and $\mathrm{PhD}$ degrees from Amirkabir University of Technology, Tehran, Iran, in 1997 and 2003, respectively. He is now Associate Professor in the Department of Polymer Engineering (Science and Research Branch) of the Islamic Azad University, Tehran, Iran. His main research interests include polymeric nanocomposites, nanoparticles, nanofibers, nanofibrous membranes, and wastewater treatment. 